Bull. Austral. Math. Soc.

VOL. $70(2004)$ [67-71]

\title{
TWISTED ALEXANDER POLYNOMIAL FOR THE LAWRENCE-KRAMMER REPRESENTATION
}

\author{
MASAAKI SUZUKI
}

\begin{abstract}
In this paper, we prove that the twisted Alexander polynomial for the LawrenceKrammer representation of the braid group $B_{4}$ is trivial. This gives an answer to the problem of whether the twisted Alexander polynomial for given faithful representations is always non-trivial.
\end{abstract}

\section{INTRODUCTION}

The twisted Alexander polynomial for finitely presentable groups was introduced by Wada in [5]. As a notable application, it was shown that the twisted Alexander polynomial can tell Kinoshita-Terasaka knot from Conway's 11-crossing knot.

In [4], the twisted Alexander polynomial for Jones representations of the braid group $B_{n}(n \geqslant 3)$ is studied. One of the main results of [4] is that the twisted Alexander polynomial for the Burau representation is not trivial for $n=3$ and trivial for $n \geqslant 4$. We know that the Burau representation is faithful for $n=3$, not faithful for $n \geqslant 5$ and the faithfulness is still open for the case $n=4$. Then it is mentioned in [4] that it would be interesting to study a relation between the faithfulness of the Burau representation and the twisted Alexander polynomial. In other words,

Problem 1.1. If a given representation is faithful, is the twisted Alexander polynomial non-trivial?

In this paper, we present the answer to this question.

Krammer constructed in [2] a representation of the braid group, which is now called the Lawrence-Krammer representation, and showed that it is faithful for $n=4$. Moreover, Bigelow [1] and Krammer [3] proved that the Lawrence-Krammer representation is faithful for all $n$. Then we may show a relation between the faithfulness of a representation and the twisted Alexander polynomial as a consequence of an explicit calculation of the twisted Alexander polynomial for the Lawrence-Krammer representation.

In this paper, we show the following. (See Section 3 for the precise statement.)

Received 30th October, 2003

The author would like to express his gratitude to Prof. Shigeyuki Morita for helpful suggestions and encouragements. This research is supported by JSPS Research Fellowships for Young Scientists.

Copyright Clearance Centre, Inc. Serial-fee code: 0004-9727/04 \$A2.00+0.00. 
THEOREM 1.2. The twisted Alexander polynomial for the Lawrence-Krammer representation of the braid group $B_{4}$ is trivial.

This gives the negative answer to Problem 1.1.

In Section 2, we briefly recall the definition of the Lawrence-Krammer representation of the braid group $B_{4}$. In Section 3, the twisted Alexander polynomial of $B_{4}$ is computed and we prove Theorem 1.2.

\section{LAWRENCE-KRAMMER REPRESENTATION OF $B_{4}$}

Let $B_{n}$ be the braid group of $n$ strings, $B_{n} \rightarrow \mathbb{Z} \simeq\langle x\rangle$ the Abelianisation and $L K$ the Lawrence-Krammer representation

$$
L K: B_{n} \longrightarrow G L\left(n(n-1) / 2 ; \mathbb{Z}\left[q^{ \pm 1}, t^{ \pm 1}\right]\right) .
$$

In this paper, we treat the case $n=4$, and we discuss the definition of the braid group and the Lawrence-Krammer representation for only this case. The braid group $B_{4}$ admits the presentation:

$$
B_{4}=\left\langle\sigma_{1}, \sigma_{2}, \sigma_{3} \mid \sigma_{1} \sigma_{2} \sigma_{1}=\sigma_{2} \sigma_{1} \sigma_{2}, \sigma_{2} \sigma_{3} \sigma_{2}=\sigma_{3} \sigma_{2} \sigma_{3}, \sigma_{1} \sigma_{3}=\sigma_{3} \sigma_{1}\right\rangle .
$$

The Lawrence-Krammer representation of $B_{4}$ is defined as follows (see $[\mathbf{1}, \mathbf{2}, \mathbf{3}]$ for general cases):

$$
\begin{gathered}
L K\left(\sigma_{1}\right)=\left(\begin{array}{cccccc}
t q^{2} & 0 & 0 & 0 & 0 & 0 \\
t q(q-1) & 0 & 0 & q & 0 & 0 \\
t q(q-1) & 0 & 0 & 0 & q & 0 \\
0 & 1 & 0 & 1-q & 0 & 0 \\
0 & 0 & 1 & 0 & 1-q & 0 \\
0 & 0 & 0 & 0 & 0 & 1
\end{array}\right), \\
L K\left(\sigma_{2}\right)=\left(\begin{array}{cccccc}
1-q & q & 0 & 0 & 0 & 0 \\
1 & 0 & 0 & t q^{2}(q-1) & 0 & 0 \\
0 & 0 & 1 & t q(q-1)^{2} & 0 & 0 \\
0 & 0 & 0 & t q^{2} & 0 & 0 \\
0 & 0 & 0 & t q(q-1) & 0 & q \\
0 & 0 & 0 & 0 & 1 & 1-q
\end{array}\right), \\
L K\left(\sigma_{3}\right)= \\
\left.\begin{array}{cccccc}
1 & 0 & 0 & 0 & 0 & 0 \\
0 & 1-q & q & 0 & 0 & 0 \\
0 & 1 & 0 & 0 & 0 & t q^{3}(q-1) \\
0 & 0 & 0 & 1-q & q & 0 \\
0 & 0 & 0 & 1 & 0 & t q^{2}(q-1) \\
0 & 0 & 0 & 0 & 0 & t q^{2}
\end{array}\right) .
\end{gathered}
$$




\section{Twisted Alexander polynomial}

In this section, we compute the twisted Alexander polynomial. All notations are the same as ones used in [4], unless we state otherwise.

First, we obtain a denominator in the twisted Alexander polynomial by an explicit calculation.

LEMMA 3.1 .

$$
\operatorname{det}\left(I_{6}-x L K\left(\sigma_{3}\right)\right)=(1-x)^{3}(1+q x)^{2}\left(1-q^{2} t x\right) .
$$

Next, we calculate a numerator in the twisted Alexander polynomial. In our case, we have the $18 \times 12$-matrix $M_{3}$ which is obtained from the Alexander matrix removing the third column. The numerator which we need is the greatest common divisor of $\operatorname{det} M_{3}^{I}$ for all the choices of the indices $I$. Here $I=\left(i_{1}, i_{2}, \ldots, i_{12}\right)$ and $M_{3}^{I}$ denotes the square matrix consisting of the $i_{k}$-th rows of the matrix $M_{3}$, where $1 \leqslant i_{1}<\cdots<i_{12} \leqslant 18$.

LEMma 3.2. For any index $I$, $\operatorname{det} M_{3}^{I}$ has a common divisor $(1-x)^{3}(1+q x)^{2}$ $\left(1-q^{2} t x\right)$

Proof: For a given $18 \times 12$-matrix $A$, we denote by $A\left(i ; a_{1}, \ldots, a_{12}\right)$ the matrix obtained from $A$ adding $a_{j}$ times the $j$-th column to the $i$-th column. We note that

$$
\operatorname{det} A\left(i ; a_{1}, \ldots, a_{12}\right)^{I}=\left(1+a_{i}\right) \operatorname{det} A^{I} .
$$

1. First, we consider

$$
M^{(1)}=M_{3}\left(4 ;-1+q^{2} t, p, p, 0,1,0,0,0,0,0,0,0\right),
$$

where $p=-1-q t+q^{2} t$. Then we can take a term $1-x$ as a common divisor from the fourth column. Next, we observe

$$
M^{(2)}=M^{(1)}\left(12 ; 0,0,0,0,0,0, q^{2}, p q,(1-q)^{2} q t,-1+q^{2} t, p, 0\right)
$$

and

$$
M^{(3)}=M^{(2)}\left(8 ;-1+q^{2} t,(-1+q) q t,(-1+q) q t, 0,0,0,-q, 0,0,0,0,0\right) .
$$

Therefore the eighth and the twelfth columns have common divisors $1-x$ and $\operatorname{det} M_{3}^{I}$ has a divisor $(1-x)^{3}$ for any index $I$.

2. Similarly, it can be considered

$$
M^{(4)}=M_{3}\left(12 ; 0,0,0,0,0,0, q^{2}, p q^{2},-1+q^{3} t-q^{4} t+p q,-q^{2}(1+q t),-p q, 0\right)
$$

and

$$
M^{(5)}=M^{(4)}\left(5 ; 0,-q^{2}, q,-q, 0,0,-q^{2},-q^{2}, 1+q, 0,0,0\right) .
$$

Then the fifth and the twelfth columns have common divisors $1+q x$ and $\operatorname{det} M_{3}^{l}$ has a divisor $(1+q x)^{2}$ for any index $I$. 
3. Finally, we set

$$
\begin{aligned}
M^{(6)}= & M_{3}\left(12 ; 0, q^{3} t(1-q)\left(1-q^{2} t\right)\right. \\
& q^{2} t(-1+q)\left(1-q^{2} t+q^{4} t^{2}+p q\right), q^{2} t(1-q)\left(1-q^{2} t\right) \\
& q t(-1+q)\left(1-q^{2} t+q^{4} t^{2}+p q\right),(1+q t)\left(1-q^{2} t\right)^{2} \\
& (1-q) q^{4} t,(-1+q) q^{4} t^{2}, q^{2} t(-1+q)\left(1-q-q t+q^{4} t^{2}\right) \\
& \left.0, q\left(1+q t-q^{2} t\right)\left(1-q^{3} t^{2}\right),\left(1-q-q^{2} t\right)\left(1-q^{3} t^{2}\right)\right)
\end{aligned}
$$

The twelfth column of $M^{(6)}$ has a common divisor $1-q^{2} t x$. We need to note that the determinant of this matrix $M^{(6) I}$ is different from that of $M_{3}^{I}$. More precisely,

$$
\operatorname{det} M^{(6) I}=\left(1+\left(1-q-q^{2} t\right)\left(1-q^{3} t^{2}\right)\right) \operatorname{det} M_{3}^{I} .
$$

However, the greatest common divisor of two polynomials $1+\left(1-q-q^{2} t\right)\left(1-q^{3} t^{2}\right)$ and $1-q^{2} t x$ is a unit, that is, they are relatively prime. This deduces that $\operatorname{det} M_{3}^{I}$ has a divisor $1-q^{2} t x$ for any index $I$. Then it completes the proof.

LEMMA 3.3. There exist indices $I_{1}, I_{2}$ such that

$$
\operatorname{gcd}\left(\operatorname{det} M_{3}^{I_{1}}, \operatorname{det} M_{3}^{I_{2}}\right)=(1-x)^{3}(1+q x)^{2}\left(1-q^{2} t x\right) .
$$

Proof: We select

$$
\begin{gathered}
I_{1}=(1,2,3,4,5,6,7,8,9,10,11,12), \\
I_{2}=(2,3,4,5,6,7,9,10,11,12,15,17)
\end{gathered}
$$

and calculate $\operatorname{det} M_{3}^{I_{1}}, \operatorname{det} M_{3}^{I_{2}}$ explicitly, then we get the conclusion.

The above two lemmas deduce that $\operatorname{det} M_{3}^{I}$ has a common divisor $(1-x)^{3}$ $(1+q x)^{2}\left(1-q^{2} t x\right)$ and does not have any other common divisor, then the numerator is settled. It follows by the definition that

THEOREM 3.4. The twisted Alexander polynomial $\Delta_{B_{4}, L K}(x)$ for the LawrenceKrammer representation with the Abelianisation $B_{4} \rightarrow \mathbb{Z} \simeq\langle x\rangle$ is given by

$$
\Delta_{B_{4}, L K}(x)=1
$$

REMARK 3.5. The twisted Alexander polynomial for the Lawrence-Krammer representation is not always trivial for $n$. In fact, we get $\Delta_{B_{3}, L K}(x)=1+q^{3} t x^{3}$ by an easy calculation. 


\section{REFERENCES}

[1] S. Bigelow, 'Braid groups are linear', J. Amer. Math. Soc. 14 (2001), 471-486.

[2] D. Krammer, 'The braid group $B_{4}$ is linear', Invent. Math. 142 (2000), 451-486.

[3] D. Krammer, 'Braid groups are linear', Ann. of Math. 155 (2002), 131-156.

[4] T. Morifuji, 'Twisted Alexander polynomial for the braid group', Bull. Austral. Math. Soc. 64 (2001), 1-13.

[5] M. Wada, 'Twisted Alexander polynomial for finitely presentable groups', Topology 33 (1994), 241-256.

Graduate School of Mathernatical Sciences

University of Tokyo

3-8-1 Komaba, Meguro

Tokyo 153-8914

Japan

e-mail: macky@ms.u-tokyo.ac.jp 